\title{
¿Existe relación entre enfermedad cardiovascular y periodontitis?
}

\author{
TEJERINA LOBO JM ${ }^{\mathrm{a}}$ * \\ CUESTA FRECHOSO $S$ * \\ MENÉNDEZ COLLAR M** \\ SICILIA FELECHOSA A*
}

\author{
Tejerina Lobo JM, Cuesta Frechoso S, Menéndez Collar M, Sicilia \\ Felechosa $A$. ¿Existe relación entre enfermedad cardiovascular y \\ periodontitis?. Av Periodon Implantol. 2003; 15, 3: 113-119.
}

\begin{abstract}
RESUMEN
La hipótesis infecciosa de la aterosclerosis ha permitido un rápido avance en el conocimiento sobre los mecanismos patogénicos de la enfermedad coronaria. Más aun, el concepto de la enfermedad coronaria como una enfermedad inflamatoria ha abierto nuevos caminos para la investigación en el campo de la cardiopatía isquémica.

Estudios recientes han sugerido que el empleo de marcadores de inflamación ayudaría en la identificación de pacientes con alto riesgo de padecer eventos cardiovasculares.

También los estudios epidemiológicos han implicado a la periodontitis como un factor de riesgo para el desarrollo de la enfermedad cardiovascular. La enfermedad periodontal proporciona una fuente de bacterias o de sus productos, y citoquinas de respuesta hiperinflamatoria del huésped por largos periodos de tiempo.

Dada la cronicidad de la enfermedad cardiovascular y de la enfermedad periodontal proba-

blemente comparten algunos caminos de común etiología.
\end{abstract}

\section{PALABRAS CLAVE}

Enfermedad cardiovascular / etiología, periodontitis, inflamación, infección

\section{INTRODUCCIÓN}

El infarto de miocardio representa uno de los grandes problemas dentro de la salud pública (1).

La cardiopatía isquémica es la causa principal de muerte en todo el mundo (cerca de 7.5 millones de personas cada año), aproximadamente el $14 \%$ de todas las muertes en todo el mundo (2). Así pues, las diversas estrategias terapéuticas pueden tener un gran impacto en el número de vidas salvadas.

El proceso patológico subyacente en la mayoría de los casos de enfermedad cardiovascular es la ateroesclerosis, proceso que se caracteriza por el desarrollo de placas fibrograsas en el árbol coronario. Las lesiones de estría grasa aparecen tempranamente, avanzan desarrollando una disfunción endotelial y progresan a formas más graves causando lesiones obstructivas en aquellas circunstancias en las cuales las placas obstruyen más del $75 \%$ de la luz del vaso. En aquellos sujetos que desarrollan la enfermedad por complicación de la placa, esta puede evolucionar hacia la muerte, recurrir, estabilizarse o regresar. El substrato añadido a la placa complicada y que ha sido evidenciado tanto en estudios angiográficos como postmorten es la presencia del trombo asociado a la erosión o rotura de la placa, hallazgo que está presente en más del $75 \%$ de las arterias coronarias de los pacientes que fallecen por cardiopatía isquémica (3).

Haciendo referencia a la patogenia de la enfermedad cardiovascular son numerosas las incógnitas que quedan por resolver y sólo se ha establecido que el taba- 
quismo y ciertas enfermedades como diabetes mellitus, hipertensión arterial e hipercolesterolemia, favorecen el desarrollo de la ateroesclerosis, constituyendo los denominados factores de riesgo vascular (4, 5, $6,7)$. Estudios experimentales, epidemiológicos y de intervención han mostrado una clara evidencia de la existencia de una asociación multicausal entre estos diversos factores. Hoy en día, parece que la existencia de un componente genético importante favorece la susceptibilidad de ciertos individuos a padecer ateroesclerosis y enfermedad coronaria.

Dichos factores, no obstante no justifican plenamente las diversas manifestaciones y distintos grados de la enfermedad en pacientes con factores de riesgo similares, ni dichos factores acontecen en todos los casos de ateroesclerosis (8).

En la actualidad se acepta que la inflamación tiene un papel fundamental en la patogenia de la ateroseclerosis, siendo el monocito una de las células fundamentales de este proceso. Fruto de este conocimiento nace el interés de considerar que determinadas infecciones pudieran actuar de forma sinérgica con los factores de riesgo vascular promoviendo la formación y el desarrollo de las placas de ateroma.

Han sido numerosos los microorganismos estudiados en relación con los procesos de ateroesclerosis, principalmente el herpesvirus, Helicobacter pylori y Chlamydia pneumoniae.

Se han explicado distintos mecanismos por los que la infección vírica podría favorecer la ateroesclerosis: los virus podrían ser capaces de inducir una proliferación monoclonal de músculo liso en la pared arterial (9). Asimismo, la infección crónica daría lugar a una respuesta inmunológica con la infiltración celular consecuente que produciría el mismo resultado (10). Sin embargo, la hipótesis de que el Citomegalovirus sea causa de ateroesclerosis es controvertida (11).

En cuanto al papel de las bacterias se han relacionado a través de estudios epidemiológicos principalmente dos: $H$. pylori, bacteria gramnegativa relacionada con la úlcera péptica, gastritis crónica y ciertos tumores gástricos; y C. pneumoniae, una bacteria intracelular gramnegativa que es causa reconocida de infecciones del tracto respiratorio superior e inferior (neumonía, bronquitis, sinusitis) y que se ha relacionado también con otras entidades como sarcoidosis o asma bronquial.

Respecto al mecanismo por el que H. pylori y C. pneumoniae podrían favorecer la ateroesclerosis existen trabajos $(12,13)$ que sugieren que la infección por estos microorganismos favorecería la producción de una Ig E específica, y ésta, a su vez, estimularía la pro- ducción de interleuquina 6 y citoquinas con importantes propiedades proinflamatorias y procoagulantes, lo que podría influir en la aparición de síndromes coronarios agudos.

En el caso de C. pneumoniae se ha llegado a cultivar el microorganismo a partir de placas de ateroma y dada la notable cantidad de evidencias científicas a favor de la relación, se han iniciado estudios prospectivos para determinar la influencia que ciertos antibióticos eficaces en el tratamiento de la infección crónica por C. pneumoniae pueden tener en la evolución de la enfermedad coronaria ateroesclerótica. Los resultados obtenidos hasta el momento, aunque no concluyentes, sí parecen prometedores pudiendo tener en breve un importante papel los antibióticos dentro del tratamiento de la cardiopatía isquémica.

En la última década se ha hecho aparente el importante papel que desempeña la inflamación en la aterogénesis y las manifestaciones clínicas de la enfermedad coronaria. Las denominadas placas activas o vulnerables, que son responsables de la aparición de eventos coronarios agudos, como el infarto de miocardio y la angina inestable, contienen numerosas células inflamatorias. El papel patogénico de la inflamación está aceptado en el momento actual; sin embargo, aún queda por clarificar el mecanismo responsable del proceso inflamatorio. Sí se ha comprobado que niveles altos de proteínas reactivas en fase aguda como la proteína $\mathrm{C}$ reactiva son marcadores de riesgo para la ateroesclerosis, y ésta es una respuesta a un proceso infeccioso como pudiera ser la enfermedad periodontal (7).

Varios estudios prospectivos bien diseñados han demostrado una asociación entre los niveles altos de proteína C reactiva y el aumento de la prevalencia de infarto de miocardio y accidentes cerebrovasculares. Esta asociación ocurre en distintos grupos de individuos: individuos sanos, ancianos, fumadores, pacientes con angina de pecho y pacientes que han tenido un infarto de miocardio previo. Se puede reducir este riesgo mediante tratamiento previo con ácido acetilsalicílico.

Los niveles altos de proteína $\mathrm{C}$ reactiva son posiblemente un marcador de los procesos inflamatorios que tienen que ver con la aterogénesis, pero también pueden indicar la presencia de infecciones crónicas que no son aparentes desde el punto de vista clínico. También pueden reflejar el grado de isquemia, y no solamente el grado de inflamación de una placa, o el grado de actividad de citocinas proinflamatorias no asociadas a una placa en particular. Recientemente se ha sugerido que la proteína $\mathrm{C}$ reactiva puede desempeñar por sí misma un papel patogénico en la ateros- 
clerosis a través de la activación del sistema de complemento.

La relación demostrada por diversos estudios entre los niveles altos de proteína $\mathrm{C}$ reactiva, el fibrinógeno y la eritrosedimentación y el pronóstico de pacientes con angina inestable confirman la importancia de los procesos inflamatorios (14).

Evidencias recientes indican que existe un estímulo antigénico crónico en pacientes con enfermedad coronaria y que éste podría ser causado por agentes infecciosos (15). Entre éstos, C. pneumoniae parece ser en el momento actual, el agente más probable (2); aunque no se descarta, sin embargo, el posible papel de otros microorganismos patógenos tales como los que se encuentran en la cavidad oral: Porphyromonas gingivalis, Actinobacillus actinomycetecomitans, Bacteroides forsythus, Prevotella intermedia, así como por Virus Coxsackie.

Varios mecanismos han sido postulados para explicar de qué modo las infecciones crónicas pueden causar enfermedad coronaria. Estudios piloto, en los que se han utilizado antibióticos macrólidos, han sugerido que estos agentes pueden mejorar el pro- nóstico de pacientes con episodios coronarios agudos y también en casos crónicos. Aunque es todavía un asunto sin clarificar, la hipótesis infecciosa de la aterosclerosis ha permitido un avance de la patogenia de la enfermedad coronaria. (16) (Tabla 1)

La visión periodontal del problema ha enunciado el término periodontitis-aterosclerosis (PAS) El síndrome PAS se propone como un nuevo término de diagnóstico para describir esta condición en estos pacientes.

Parece ser que la periodontitis es un factor de riesgo como los lípidos en sangre, el índice de masa corporal y la presión sanguínea para la enfermedad cardiovascular. Sólo la historia familiar es un factor de riesgo más potente. La importancia de historia familiar para el riesgo podría sugerir que hay genes de susceptibilidad que se heredan y ponen a ciertos individuos al riesgo para ataque cardíaco. Al igual que la periodontitis.

Un individuo PAS de alto riesgo cardiovascular en relación a magnitud periodontal sería el que tuviera niveles de pérdida de inserción de más de $3 \mathrm{~mm}$ en más del $60 \%$ (17).

TABLA 1.

\section{MODELO DE INTERACCIÓN ENTRE ENFERMEDAD PERIODONTAL- ENFERMEDAD CARDIOVASCULAR}

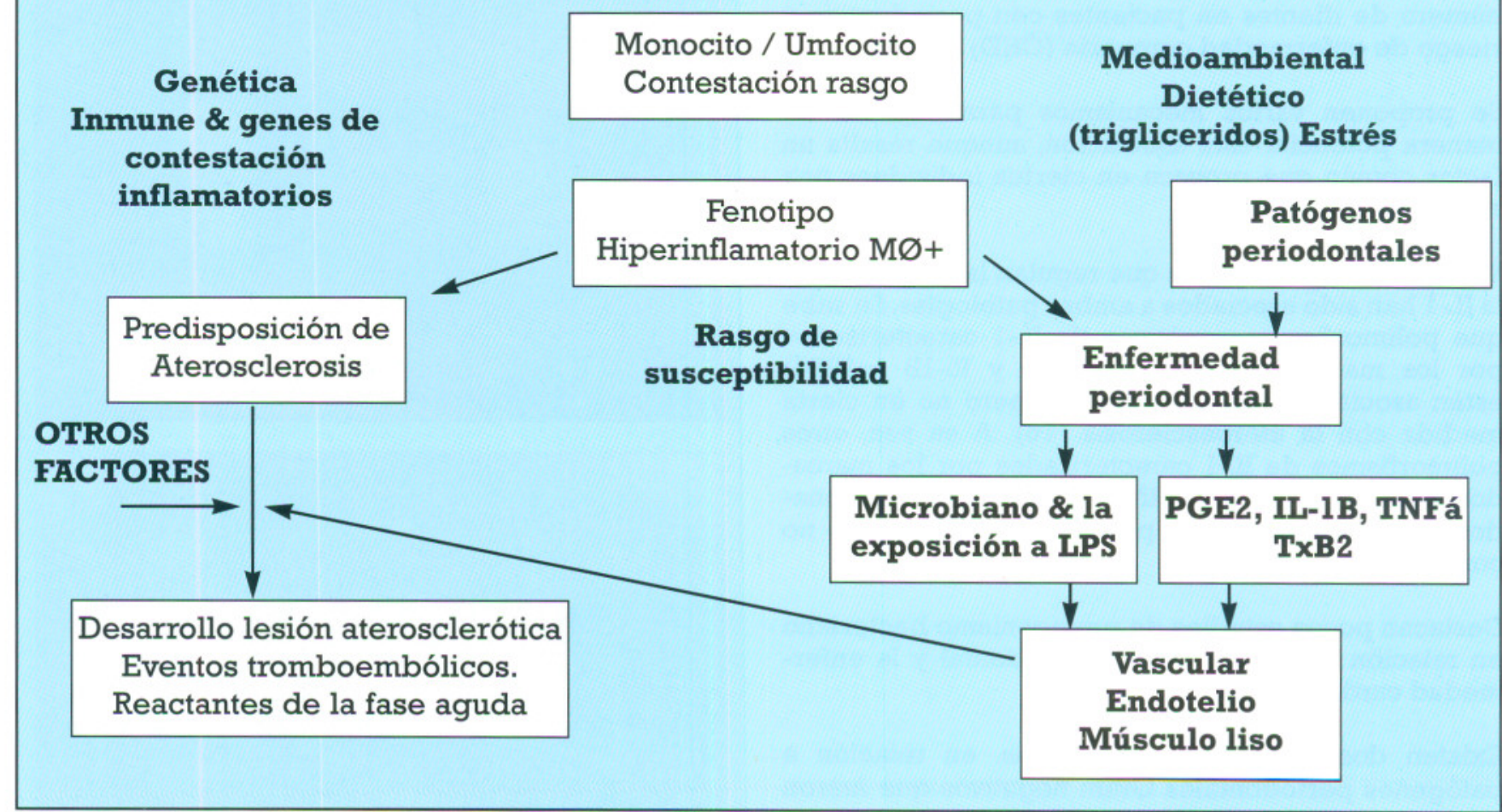

Tomado de Annals of Periodontology, Julio 1998; Vol. 3- $n^{\circ} 1$ 


\section{AVANCES}

Volumen 15 - No 3 - Diciembre 2003

Conceptualmente, esto es soportado por una carga microbiana mayor que aumentaría el riesgo cardiovascular. Por sí sola, la placa bacteriana no confiere riesgo para los PAS, pero si la periodontitis no está controlada el riesgo aumenta con más cantidad de placa. Estos hallazgos sugieren que la condición periodontal crearía una herida anatómica por la que los organismos orales y los productos inflamatorios pudieran ocasionar problemas sistémicos. Así, el periodonto parece representar una barrera que impide que los efectos injuriosos de la microbiota oral causen daño cardiovascular.

En la actualidad, múltiples estudios han puesto de manifiesto una asociación epidemiológica entre la enfermedad periodontal y la enfermedad cardiovascular.

Beck y Offenbacher evalúan 9 estudios epidemiológicos relacionando la enfermedad periodontal (EP) y la enfermedad cardiovascular (ECV). Tienen el inconveniente de haberse realizado en distintos países con años de seguimiento no similares, que van desde 6 a 21 años (18).

Sólo por el tamaño de las muestras (que es muy elevado) se puede considerar una asociación bajamoderada entre EP y ECV.

El estudio de Hujoel es crítico con esta apreciación, manifestando que el riesgo de enfermedad coronaria no aumentó substancialmente con la severidad de periodontitis. Ninguna relación se identificó entre el número de dientes en pacientes con periodontitis y riesgo de enfermedad coronaria (CHD) (19).

Se proponen varios mecanismos para explicar de manera potencial esta asociación, aunque resalta un factor común que provoca en ciertos individuos una respuesta inflamatoria.

Variaciones en los genes que regulan la respuesta de la IL-1 han sido asociados a ambas patologías. Se sabe que polimorfismos genéticos de IL-1 caracterizados por los marcadores IL-la (+4845) y IL-1B (+3954) están asociados con periodontitis pero no en cierta medida con la ateroesclerosis (16). A su vez, otros polimorfismos de IL-l caracterizados por los marcadores IL-1B (-511) y IL-1RN (+2018) están relacionados con la formación de placas de ateroma pero no con la periodontitits (20).

Destacan pocos estudios de protagonismo bacteriano en relación a la enfermedad periodontal y la enfermedad cardiovascular.

Existen dos estudios preeliminares, en relación a patógenos periodontales Gram negativos que fueron encontrados en placas de ateroma (21).

\section{CASO 1 - C.A.A.G.}

Varón 35 años.

Fumador $30 \mathrm{c} /$ día.

Problema cardiovascular hace 6 años: Infarto.

Cálculo supra / subging. muy abundante.

Periodonto fino. Reborde alveolar normal.

Percial resina inf. Nada en max. sup.

Ultima tartrectomía hace 2 años.

Dientes presentes en boca $=9$.

Movilidades: 33 en : $28-34$ Sondajes: $4-9 \mathrm{~mm}$

${ }^{\circ} 2+$ en : 43

2 - en : 35

${ }^{\circ} 1+$ en : 17-44

${ }^{\circ} 1$ - en : 37-46

$\mathrm{IH}=100 \% \mathrm{IP}=100 \%$

Pronóstico grave a corto plazo para todos los dientes.

Estudio microbiológico. Presencia de P. gingivalis.
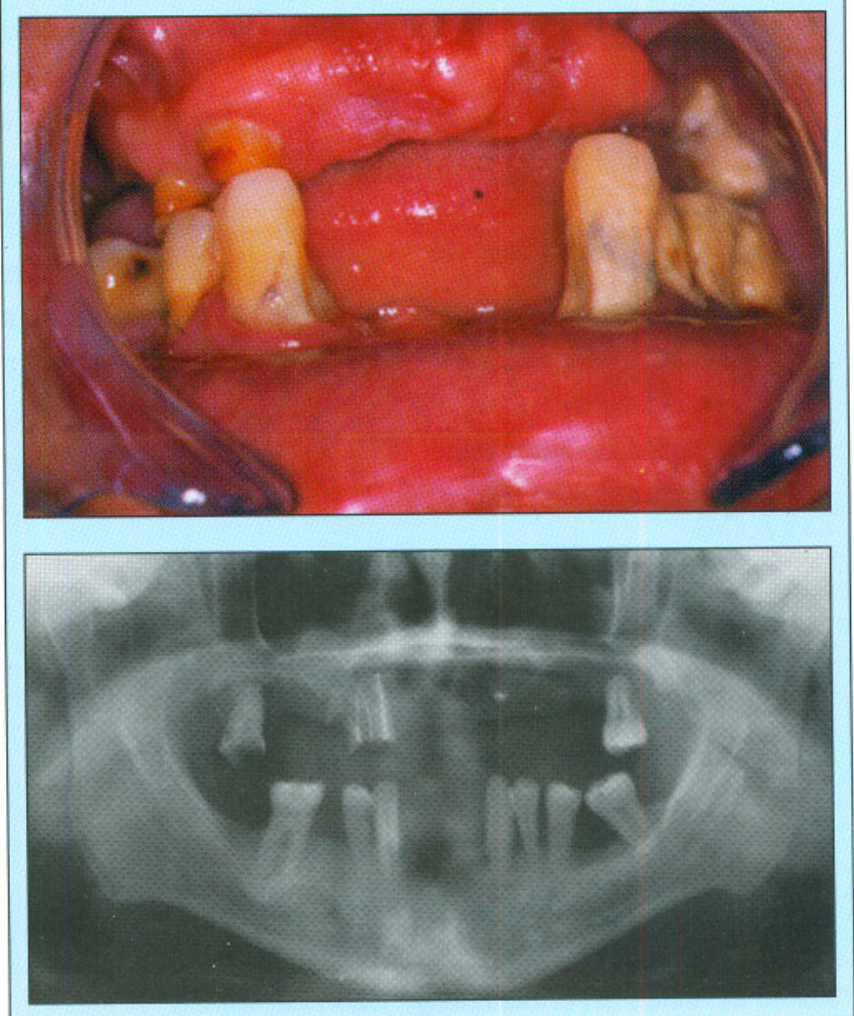


\section{CASO 2 - L.A.F.}

Varón 52 años.

Ex-fumador $20 \mathrm{c} /$ día hace 8 años.

Problema cardiovascular hace 8 años: Infarto.

Cálculo supra / subging. abundante.

Periodonto normal. Reborde alveolar ancho.

Mordida borde a borde.

Ultima tartrectomía hace 5 años.

Dientes presentes en boca $=27$.

Movilidades: ${ }^{\circ} 3$ en : 21

2 en : $42-45$

'2- en : 41-47

${ }^{\circ} 1+$ en : 15-25-38-37-32-44

${ }^{\circ} 1$ en : 13-12-24-35

${ }^{\circ} 1-$ en 18-17-16-14-11-21-23-27-

43-48

Estudio microbiológico. Presencia de P. gingivalis.
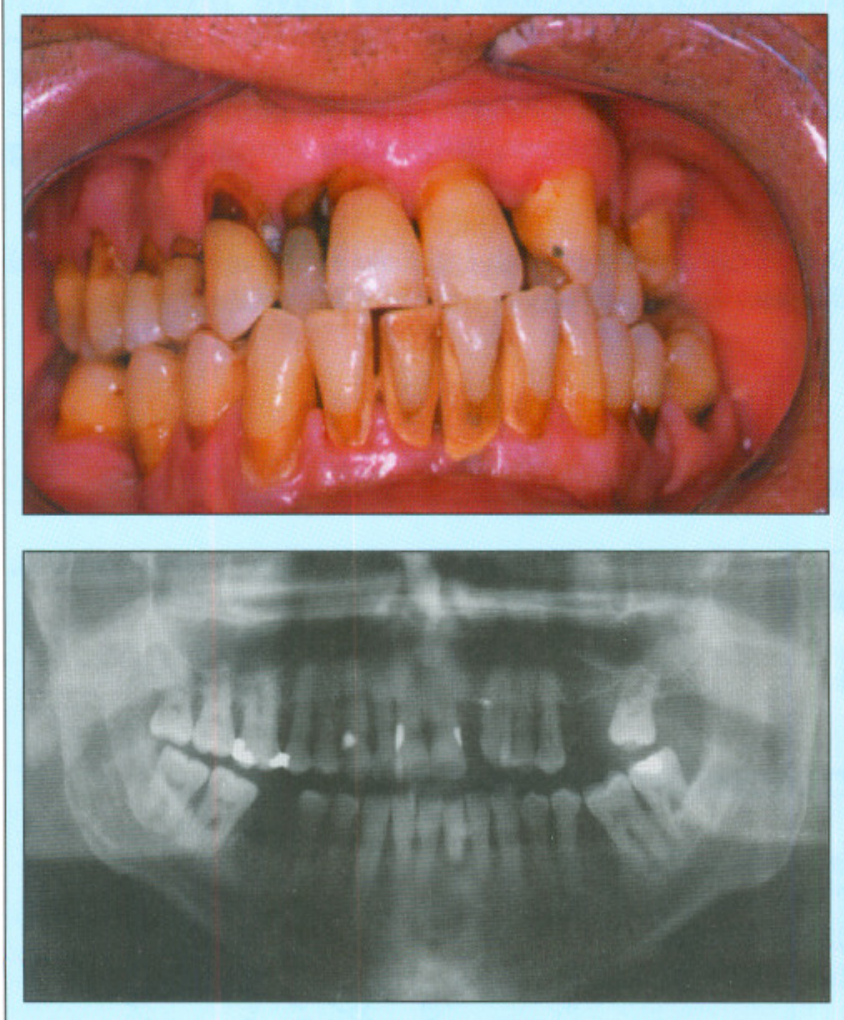

El primero de ellos se describen:

Cincuenta especímenes quirúrgicos adquiridos en endoarteroctomía de carótida para valorar Chlamydia, citomegalovirus y patógenos periodontales.

Veintidós de estos especímenes eran positivos para patógenos periodontales.

Eran positivos el $26 \%$ para B. forsythus, $25 \%$ eran positivos para $P$. gingivalis, $13 \%$ eran positivos para $A$. actinomycetemcomitans, $16 \%$ eran positivos para $P$. intermedia y $2 \%$ era positivo para C. rectus.

Las muestras anatomo-patológicas en 88 (95\%) de los 93 y en 62 especimenes de endoarterectomía (94\%) de 66, eran positivos para uno o los microorganismos más designado.

En otro estudio del mismo grupo, los especimenes quirúrgicos eran 93 ateromas de carótida, y $58 \%$ de los especímenes eran positivos para algún patógeno periodontal (22).

Los resultados de Cuttler en 1999 indican la presencia de una relación significativa entre la periodontitis, hiperlipidemia, y anticuerpos de suero contra el lipopolisacárido (LPS) de P. gingivalis. Fue un estudio realizado en una muestra de 26 pacientes con un grupo control de 25. Además, estos estudios indican que los niveles elevados de triglicéridos pueden modular una relación entre IL - 1B y el aumento de producción estimulado por LPS de $P$. gingivalis PMNs (23).

\section{RIESGO COIMPARTIDO: TABACO}

Fumar tiene propiedades patogénicas potenciales que pueden producir aterosclerosis y enfermedad periodontal, y fumar se reconoce como un factor de riesgo compartido. Un punto de énfasis es que los estilos de vida de los fumadores pueden ser diferentes, y su habilidad para ignorar los mensajes de salud públicos puede predisponerlos a enfermedad cardiovascular debido a una dieta pobre, falta de ejercicio y otras prácticas de estilo de vida no saludables. Los mismos individuos también pueden estar predispuestos a ignorar consejos de salud dental que pueden resultar en una falta de higiene oral y así desarrollar una enfermedad periodontal no controlada $(24,25)$.

Desde un punto de vista biológico, fumar puede tener un efecto adverso en la función de los fibroblastos y disfunción a la quimiotaxis y la fagocitosis de los neutrófilos, induce a una vasoconstricción periférica. 
Por lo tanto, las observaciones presentes identifican fumar como impacto negativo en la salud general y proporcionan evidencia extensa de que el fumar tabaco debe ser un riesgo evitable.

Los estudios muestran una condición de salud periodontal inferior asociada con fumar.

En relación a la periodontitis fue evidenciada una frecuencia significativamente mayor de sondajes activos y una reducción significativamente mayor de altura del hueso periodontal en fumadores actuales comparando a los no fumadores.

Fumadores anteriores que han dejado de fumar tienen una condición de salud periodontal mejor que los fumadores actuales, aunque peor que los no fumadores.

Un estudio reciente indica que la periodontitis puede asociarse con infarto agudo de miocardio (26).

Basándonos en este estudio realizado en Turquía nos involucramos en estudiar periodontalmente a los pacientes ingresados con infarto agudo de miocardio que estén en un campo de edad entre 30-60 años, en la principal institución hospitalaria de nuestra ciudad de residencia.

Hemos recogido en 35 pacientes y sus parámetros clínicos, tanto médicos como periodontales y hemos observado que desde el punto de vista preventivo sí es importante dicha relación en estos pacientes.

Ninguno de los pacientes examinados tenía conocimientos de higiene oral, su control de placa era mínimo y su estado bucal de total abandono (Casos clíni$\cos 1$ y 2$)$.

\section{CONCLUSIONES}

La enfermedad cardiovascular y la periodontitis tienen factores de riesgo en común, por lo que los estudios deben interpretarse con cautela.

Estos estudios no se diseñaron para valorar esta asociación y han sido integrados desde una perspectiva diferente.

A pesar de los factores de confusión y modificaciones de efecto, debe considerarse la enfermedad periodontal como un factor de riesgo independiente para enfermedad cardiovascular. La fuerza de asociación es baja moderada, según estudios nombrados anteriormente.
Recientes meta-análisis han mostrado las asociaciones de marcadores de suero de C. pneumoniae y $H$. pylori con la enfermedad arterial, no existiendo todavía ningún meta-análisis sobre microbiología específica periodontal.

La contribución esencial de enfermedades dentales y orales al mantenimiento de la salud general todavía no se aprecia ampliamente, y serán necesarios más estudios prospectivos y con un gran tamaño muestral para determinar la posible relación de causalidad entre enfermedades periodontales y la enfermedad cardiovascular.

\section{SUMMMARY}

The infectious theory to explain atherosclerosis has allowed researchers to advance quickly on the knowledge about pathogenia of cardiovascular disease. Even more, the concept of coronary disease as an inflammatory disease has developed new pathways for investigating the ischaemic cardiac.

Recent investigations suggest that the use of inflammatory markers may help for early detecting patients who present high risk of cardiovascular strokes appearance.

Also, epidemiological essays have revealed that periodontitis is a risk factor for cardiovascular disease to develop. Periodontitis is a source of bacterias or their wastes, and provoke a hyperinflammatory response on the hostage, liberating cytokines for long periods.

Due to chronicity of both cardiovascular and periodontal disease, they may have some similar ethilogycal ways.

\section{KEY WORDS}

Cardiovascular disease / ethiology, periodontitis, inflammation, infection.

\section{CORRESPONDENCIA}

José María Tejerina

$\mathrm{P}^{\circ}$ Begoña, $24-6^{\circ} \mathrm{B}$

33205 Oviedo

Tel.: 9855354706 


\section{BIBLIOGRAFÍA}

1. Uemura $\mathrm{K}$, Pisa Z. Trends in cardiovascular disease mortality in industrialized countries since 1950. World Health Stat Q 1988; 41: 155-78.

2. Saikku P, Leinoen M, Mattila K. Serologic evidence of an association of a novel Chlamydia, TWAR, with chronic coronary heart disease and acute myocardial infarction. Lancet. 1998; 2: 983-6.

3. Davids MJ, Thomas A. Thrombosis an acute coronary artery lesions in sudden cardiac ischaemic death. $\mathrm{N}$ Engl J Med 1984; 310: 1137-40.

4. Kannel WB, Dawber TR, Kagan A, Revotskie N, Stokes J. Factors of risk in the development of coronary heart disease. Six year follow-up experience: The Framingham study. Ann Inter Med 1961; 55: 333-40.

5. Keys A. Coronary heart disease in seven countries. Circulation 1970; 41: 1-199.

6. Psaty B, Koepsell TD, Manolio TA, Longstreth WT, Wagner EH. Risk ratios and risk differences in estimating the effect of risk factors for cardiovascular disease in the elderly. J Clin Epidemiol 1990; 3, 961-70.

7. Lowe GD. Eiopathogenesis of cardiovascular disease: Hemostasis, thrombosis and vascular medicine. Ann Periodontol 1998; 3: 127-41.

8. Gorden T, García-Palmieri MR, Kagen A, Kannel WB, Schiffman J. Differences in coronary heart disease in Framingham, Honolulu and Puerto Rico. J Chron Dis 1974; 27: 329-44.

9. Benditt EP, Benditt JM. Evidence for a monoclonal origin of human atherosclerotic plaques. Proc Natl Acad Sci USA 1973; 70: 1753-6.

10. Kaner RJ, Hajjar DP. Viral genes and atherogenesis 1992; 62-81.

11. Adler SP, Hur JK, Wang JB, Vetrovee GW. Prior infection with cytomegalovirus is not a major risk factor for angiographically demonstrated coronary artery atheroesclerosis. J Infect Dis 1998; 177: 209-12.

12. Leinonen M. Pathogenetic mechanisms and epidemiology of Chlamydia pneumoniae. Eur Heart J 1993; 14: 57-61.
13. Aceti A, Mazzacurati G, Amendolea MA, Pennica A, Zechini B, Trappolini L. Relation of C-Reactive protein to cardiovascular risk factors: $\mathrm{H}$ pylorimand $\mathrm{C}$ pneumoniae infections may account for most acute coronary syndromes. Br Med J 1996; 313: 428-9.

14. Kaski JC. Inflamación, infección y enfermedad coronaria: mitos y realidades. Rev Esp Cardio 2000; 53: 1311-7.

15. Fuster V, Badimon L, Badimon JJ, Chesebro JH. The pathogenesis of coronary artery disease and the acute coronary syndromes (part I). New Engl J Med 1992; 326: 24250 .

16. Kinane DF. Periodontal diseases. Contributions to cardiovascular disease: An overview of potencial mechanisms. Ann Periodontol. 1998; 3 (1): 142-50.

17. Offenbacher S. Periodontitis - atherosclerosis syndrome: an expanded model of pathogenesis. J Periodont Res $1999 ; 34: 346-52$.

18. Beck JD. Annals of Periodontology, Julio 1998; Vol. $3 n^{\circ} 1$.

19. Hujoel P. Periodontal disease and coronary heart disease risk. JAMA Septiembre 20, 2000; Vol. 284.

20. Hegele RA. The pathogenesis of atherosclerosis. Clin Chim Acta 1996; 246: 21-38.

21. Haraszthy VI, Zambon JJ, Trevisan M, Zeid M, Genco RJ. Identification of periodontal pathogens in atheromatous plaques. J Periodontol 2000; 71: 1554-61.

22. Haraszthy V.I. Periodontal pathogens in atheromas from a German population. Ann Periodontol 2001; 6: 64.

23. Cutler CW. Association between periodontitis and hyperlipidemia. Cause or effect?. J Periodontol 1999; 70: 1429-34.

24. Bergström J., Eliasson S. y Dock J. Exposure to tobacco smoking and periodontal health. J Clin Periodontol 2000; 27:61-8.

25. Kinane D. How periodontal disease may contribute to cardiovascular disease. Periodontology 2000; vol. 23: 121-6.

26. Emingil G. Association between periodontal disease and acute myocardial infarction. J Periodontol 2000; 71 : 1882-6. 
